Original article

\title{
Trajectories and determinants of functional limitations in late-life depression: A 2-year prospective cohort study
}

\author{
Sanne Wassink-Vossen ${ }^{\mathrm{a}, *}$, Rose M. Collard ${ }^{\mathrm{b}}$, Klaas J. Wardenaar ${ }^{\mathrm{c}}$, Peter F.M. Verhaak ${ }^{\mathrm{d}, \mathrm{e}}$, \\ Didi Rhebergen $^{\mathrm{f}, \mathrm{g}}$, Paul Naarding ${ }^{\mathrm{a}}$, Richard C. Oude Voshaar ${ }^{\mathrm{c}}$ \\ a Department of Old-age Psychiatry, GGNet Apeldoorn/Zutphen, The Netherlands \\ ${ }^{\mathrm{b}}$ Department of Psychiatry, Radboud University Medical Center, Nijmegen, The Netherlands \\ ' University of Groningen, University Medical Center Groningen, University Center for Psychiatry, Groningen, The Netherlands \\ d University of Groningen, University Medical Center Groningen, Department General Practice, Groningen, The Netherlands \\ e Netherlands Institute of Health Services Research, Utrecht, The Netherlands \\ ${ }^{\mathrm{f}}$ Amsterdam UMC, Vrije Universiteit, Psychiatry, Amsterdam Public Health research institute, The Netherlands \\ ${ }^{g}$ GGZ inGeest Specialized Mental Health Care, Amsterdam, The Netherlands
}

\section{A R T I C L E I N F O}

\section{Article history:}

Received 5 May 2019

Received in revised form 4 September 2019

Accepted 6 September 2019

Available online 21 September 2019

\section{Keywords:}

Depression

Functional limitations

Recovery

Ageing

\begin{abstract}
A B S T R A C T
Background: In mental health research, functional recovery is increasingly valued as an important outcome in addition to symptomatic remission.

Methods: Course types of functional limitations among depressed older patients and its relation with symptomatic remission were explored in a naturalistic cohort study (Netherlands Study of Depression in Older persons). 378 depressed older patients ( $\geq 60$ years) and 132 non-depressed persons were included. Depressive disorders were assessed with Composite International Diagnostic Interview at baseline and two-year follow-up. Functional limitations were assessed every 6 months with the World Health Organization Disability Assessment II.

Results: Depressed patients had more functional limitations compared to their non-depressed counterparts. Growth Mixture Modeling among depressed patients identified two trajectories of functional limitations, both starting at a high disability level. The largest subgroup (81.2\%) was characterized by a course of high disability levels over time. The smaller subgroup (18.8\%) had an improving course (functional recovery). After two years, the main predictor of functional recovery was the remission of depression. Among symptomatic remitted patients, female sex, higher level of education, higher gait speed, and less severe depression were associated with no functional recovery. Non-remitted patients without functional recovery were characterized by the presence of more chronic somatic diseases, a lower sense of mastery, and a higher level of anxiety.

Conclusions: 1 in 5 depressed older patients have a course with functional recovery. Combining functional and symptomatic recovery points to a subgroup of older patients that might profit from more rigorous psychiatric treatment targeted at psychiatric comorbidity and a group of frail depressed older patients that might profit from integrated geriatric rehabilitation.
\end{abstract}

(C) 2019 Elsevier Masson SAS. All rights reserved.

\section{Introduction}

Major Depressive Disorder (MDD) is common in later life with prevalence rates around 5\% [1,2]. Studies on the natural course of depressive disorders as well show that late-life depression has poor prognosis with respect to symptomatic improvement and/or remission [3-6]. In mental health research the level of functioning

\footnotetext{
* Corresponding author at: GGNet Department of Old-age Psychiatry, P.O. Box 2003, 7230 GC Warnsveld, The Netherlands.

E-mail address: s.wassink@ggnet.nl (S. Wassink-Vossen).
}

of individual patients is increasingly valued as an important outcome $[7,8]$, as recovery in daily functioning is most important from a patient perspective [9]. Functioning refers to a persons' capacity to perform activities and their ability to be in interaction with the environment [10].

Previous studies on functional outcome in depressed patients show that a substantial part of the patients with symptomatic improvement also significantly improve in their functioning $[11,12]$. However, many remitted patients do not reach the level of functioning of non-depressed controls [13], even when controlled for comorbid chronic somatic diseases and cognitive decline $[14,15]$. More knowledge on course of functional recovery 
and its determinants in late-life depression may provide new insights in what is needed in the treatment and care for older persons with depression [16].

The first objective of the present study is to compare the level of functional limitations between depressed patients and a nondepressed comparison group. We hypothesize that depressed patients are functionally more impaired compared to nondepressed persons. Secondly, we will explore which course types of functional limitations can be identified over a two-year followup of depressed older patients. Thirdly, we will explore which determinants differentiate between the specific course types we identified with and without stratification for symptomatic remission at two-year follow-up.

\section{Methods}

\subsection{Study sample}

Data were obtained from the baseline and two-year assessments of the Netherlands Study of Depression in Older Persons (NESDO), a multi-centre cohort study that examined the natural course, determinants, and consequences of depression in later life [17].

From 2007 to 2010, 378 older persons diagnosed with depression and 132 never-depressed older persons were recruited from mental health care and primary care settings in five regions in the Netherlands (total $N=510$ ). Subjects in both the depressed and the comparison group were $\geq 60$ years of age. Inclusion criteria for patients were the presence of a depressive disorder according to DSM-IV (see below). Inclusion criteria for the non-depressed comparison group were no lifetime diagnosis of any depressive disorder. Exclusion criteria for both groups were a Mini Mental State Examination score (MMSE) under 18, a primary diagnosis of dementia or insufficient command of the Dutch language. The study protocol of NESDO was approved by the ethical review boards of all participating study centers. Written informed consent was obtained from all participants, cases and controls at the start of the baseline assessment.

All participants received an extensive assessment of psychopathology, socio-demographic characteristics, physical health and physical health markers, cognitive functioning, psychosocial functioning, and life style variables at baseline and at two-year follow-up. The course of late-life depression was followed up every 6 months by means of a postal assessment including self-report questionnaires. Well-trained research assistants, mainly psychologists and mental health care nurses, conducted the interviews. All interviews were audio taped and were regularly controlled for their quality.

\subsection{Measurements}

\subsubsection{Functional limitations and functional recovery}

Functional limitations were measured every 6 months with the World Health Organization Disability Assessment (WHODAS II). This self-report questionnaire integrates an individual's level of functioning in major life domains and directly corresponds with the ICF "activity and participation" dimensions. The WHODAS II contains 36 items covering the six domains of functioning during the last 30 days: 1 . Cognition (understanding and communicating); 2. Mobility (moving and getting around); 3. Hygiene, dressing, eating and staying alone (self-care); 4. Interpersonal actions (getting along with people); 5. Work, leisure, domestic responsibilities (household activities); 6. Joining in community activities (participation in society). The items about work were omitted for the NESDO study, because most of the participants were retired from work. Total score was calculated. Subsequently, the scores were transformed into adjusted scores on a scale from 0 to 100 , where higher scores reflect greater disability [18]. Course trajectories of functional limitations over time were explored by Growth Mixture Models (see statistical analyses).

\subsubsection{Depression}

The presence of a depressive disorder in the last 6 months prior baseline measurement, i.e. MDD, minor depression or dysthymic disorder, was obtained from the Composite Interview Diagnostic Instrument (CIDI) version 2.1. The CIDI has been developed by the WHO [19], and we added some additional questions to be able to diagnose also a past-month minor depression. Remission at follow-up was defined as the absence of a depressive disorder according to the CIDI at 2-year follow up.

\subsubsection{Determinants of the trajectories of functional limitations}

As we performed an explorative study on the determinants of trajectories of functional limitations, we assessed different clusters of potential determinants, i.e. demographics, cognitive function, somatic condition, psychosocial factors, lifestyle and psychopathology.

Demographics; age, sex, and educational level were obtained from general questions from the baseline interview.

Cognitive function is operationalized in five domains based on different cognitive tests. Global cognitive function (MMSE) [20], and four specific neuropsychological tests: memory (retention score of the immediate and delayed recall on the 10 words test (Auditory Verbal Learning Test)) [21], processing speed (difference between card I and II on Stroop Color-Word test (short form), interference (interference score on the Stroop test) [22], and working memory (digit span backwards) [23].

Somatic condition:The number of chronic diseases was assessed by self-report questions about the presence of somatic diseases (cardiac diseases, cerebrovascular accident, hypertension, peripheral atherosclerosis, diabetes mellitus, chronic non-specific lung disease, liver diseases, thyroid diseases, epilepsy, intestinal diseases, arthritis/arthrosis, and cancer). The accuracy of selfreports of these diseases was shown to be adequate and independent of cognitive impairment in comparison with data obtained from general practitioners [24]. Medication use was defined as the total number of different medications (according to the Anatomical Therapeutic Chemical Classification System) that were used in the past six months and was obtained from the interview. This information was completed with a container check by the research assistants during the interview. Body Mass Index (BMI) was calculated as weight in kilograms divided by the square of height in centimeters. To assess handgrip strength, participants were asked to perform two squeezes on a dynamometer, using their dominant hand. The best performance, recorded as strength in kilograms, was used for analysis. Gait speed was measured by a six-meter walking test and expressed in seconds/finish.

Lifestyle factors: Physical activity was measured with the International physical activity questionnaire (IPAQ). According to the WHO and official scoring protocol of the IPAQ [25] we considered the IPAQ categories of moderate and high physical activity as sufficient physical activity and third category of low physical activity as insufficient physical activity. The 'moderate' category on the IPAQ is equivalent to "half an hour of at least moderate-intensity PA on most days", the former leisure timebased PA population health recommendation by the WHO. The IPAQ questions on sitting are used as an indicator of time (minutes) spent in sedentary behavior on an average day. Smoking of subjects was dichotomized into current smoker and non-smoker/former smoker. Alcohol use was measured with the Alcohol Use Disorders Identification Test (AUDIT). The sum score was used as an indicator 
of the severity of (problematic) alcohol use with higher scores indicating more alcohol use [26].

Psychosocial factors: Data of partner status and network size were obtained from a selection of the Close Person Inventory (CPI) [27]. The Mastery Scale was used to determine the sense of mastery on one's own life [28]. On this scale, lower scores (range 5-25) indicate a higher sense of mastery.

Psychopathology: The severity of depression was assessed by the 30-item Inventory of Depressive Symptomatology Self Report (IDS-SR) [29]. Anxiety severity was assessed by the Beck Anxiety Index (BAI) [30].

\subsection{Statistical analyses}

First, we compared the level of functional limitations between depressed persons $(n=378)$ and the non-depressed comparison group ( $n=132$ ) with multivariable ANOVA adjusted for potential confounders associated with both depression and functional limitations, i.e. demographics (age, sex, level of education), somatic comorbidity (number or somatic diseases) and global cognitive functioning (MMSE sum score).

As subsequent analyses on the depressed subgroup are based on prospective data, we compared study completers and noncompleters with respect to the main characteristics and potential confounders listed above.

We used Growth Mixture Models (GMM) to identify latent classes with different longitudinal trajectories of functional limitations. GMM is a technique developed to identifying distinct subpopulations or latent classes, based on trajectories of growth over time (consisting of an intercept and slope parameter) [31]. In the current analyses, linear growth was modeled and random intercepts and fixed slopes were estimated for each class. The GMM was performed in the statistical package Mplus 5.0 [32]. The optimal GMM model was identified by estimating models with increasing numbers of classes and comparing the estimated models using the Bayesian and Akaike Information Criteria (BIC and AIC), with the lowest values indicating the best model. In addition, class size was also considered in model selection with models with classes of $\leq 10$ individuals being deemed of limited use for further analyses. After the optimal model selection, persons were allocated to their most likely class based on their posterior class probabilities. All analyses were run with multiple random starts and used a robust maximum likelihood estimator. Depressed patients with less than three out of the five WHO-DAS II measures were excluded. Unfortunately, due to skewed distribution of subscale scores (high frequency of null scores), GMM models could not be adequately fitted. Therefore, only the WHO-DAS sumscore was used in these analyses.

Finally, logistic regression models with latent class status as the dependent variable were built to explore all baseline characteristics described in the method section as potential determinants of class status as well as their interaction with depression status at two-year follow-up. Because of strong interaction of our independent variables with remission of depression, these analyses were repeated stratified for symptomatic remission at two-year follow-up using multinomial logistic regression. With respect to objective 1 (not explorative) and objective 2 (explorative), our study has sufficient power, only objective 3 is limited by multiple testing. Because of the explorative nature of the third research question, an uncorrected threshold of $\mathrm{p}<0.05$ was applied here as well.

Expect for the GMM, all analyses were performed using SPSS version 22 . P-values less than 0.05 were considered statistically significant.

\section{Results}

\subsection{Patient characteristics}

Table 1 presents the main baseline characteristics for depressed and non-depressed groups separately. Depressed patients had a significantly lower level of education, lower overall cognitive functioning and more chronic somatic diseases (Table 1 ).

Adjusted for age, sex, years of education, MMSE score, and number of chronic diseases, depressed patients had a significantly higher mean (standard error) level of functional limitations compared to their non-depressed counterparts (24.9 $(\mathrm{SE}=0.6)$ versus 8.6 ( $\mathrm{SE}=1.0) ; \mathrm{F}=200.2, \mathrm{df}=484,1, \mathrm{p}<.001)$.

\subsection{Course types of functional limitations}

Of the 378 depressed patients, 65 depressed patients had less than two sum scores of the WHO-DAS II available and were excluded in the GMM analyses. The 65 patients excluded did not statistically differ from those included $(n=313)$ with respect with sex, years of education, number of chronic diseases, but were significantly older $(72.2(S D=7.7)$ versus $70.3(S D=7.3), t=2.3$, $\mathrm{df}=376, \mathrm{p}=.021)$ and scored significantly lower on the MMSE (26.5 $(\mathrm{SD}=2.7$ ) versus $28.0(\mathrm{SD}=1.7), \mathrm{t}=-5.5, \mathrm{df}=375, \mathrm{p}<.001)$.

The GMM showed that the model fit increased with each added class until a 5-class model, where estimation problems arose. However, both the 3- and 4-class models had some very small classes $(n=5)$ making them of limited use for further analyses. Therefore, the 2-class model was selected as the final model and used to allocate patients to subgroups. The plotted mean WHODAS II scores over time (Fig. 1) show that the included sample has a high mean baseline disability level and that the largest subgroup is characterized by either a stable course over time $(n=254,81.2 \%)$ and the smaller subgroup does have an improving course $(n=59$, $18.8 \%$ ). This latter class was named the "functional recovery" class.

Table 2 present the baseline characteristics of the 313 patients as well as the odds ratio by which characteristics predicts class membership. Only a higher age $(\mathrm{OR}=1.05$ [95\% CI: $1.01-1.10]$, $\mathrm{p}=.028)$ and more chronic somatic diseases $(\mathrm{OR}=1.27[95 \% \mathrm{CI}$ : $1.01-1.61], \mathrm{p}=.040$ ) significantly predicted no functional recovery after two years.

Table 1

Demographics and clinical characteristics of (non-) depressed older adults.

\begin{tabular}{|c|c|c|c|}
\hline Characteristics & $\begin{array}{l}\text { Non-depressed persons } \\
(\mathrm{N}=132)\end{array}$ & $\begin{array}{l}\text { Depressed patients } \\
(\mathrm{N}=378)\end{array}$ & Statistics \\
\hline Age (years), mean (SD) & $70.1(7.2)$ & $70.7(7.4)$ & $\mathrm{t}=-0.9, \mathrm{df}=508, \mathrm{p}=.370$ \\
\hline Gender, \% female & $61.4 \%(81 / 132)$ & $66.1 \%(250 / 378)$ & $\mathrm{Chi}^{2}=1.0, \mathrm{df}=1, \mathrm{p}=.322$ \\
\hline Level of education (years), mean (SD) & $12.6(3.5)$ & $10.4(3.4)$ & $\mathrm{t}=5.8, \mathrm{df}=508, \mathrm{p}<.001$ \\
\hline Global cognitive functioning, mean (SD) & $28.3(1.6)$ & $27.7(2.0)$ & $\mathrm{t}=3.4, \mathrm{df}=507, \mathrm{p}<.001$ \\
\hline Number chronic diseases, mean (SD) & $1.5(1.1)$ & $2.1(1.5)$ & $\mathrm{t}=-4.5, \mathrm{df}=507, \mathrm{p}<.001$ \\
\hline
\end{tabular}

Abbreviations: SD, standard deviation. 

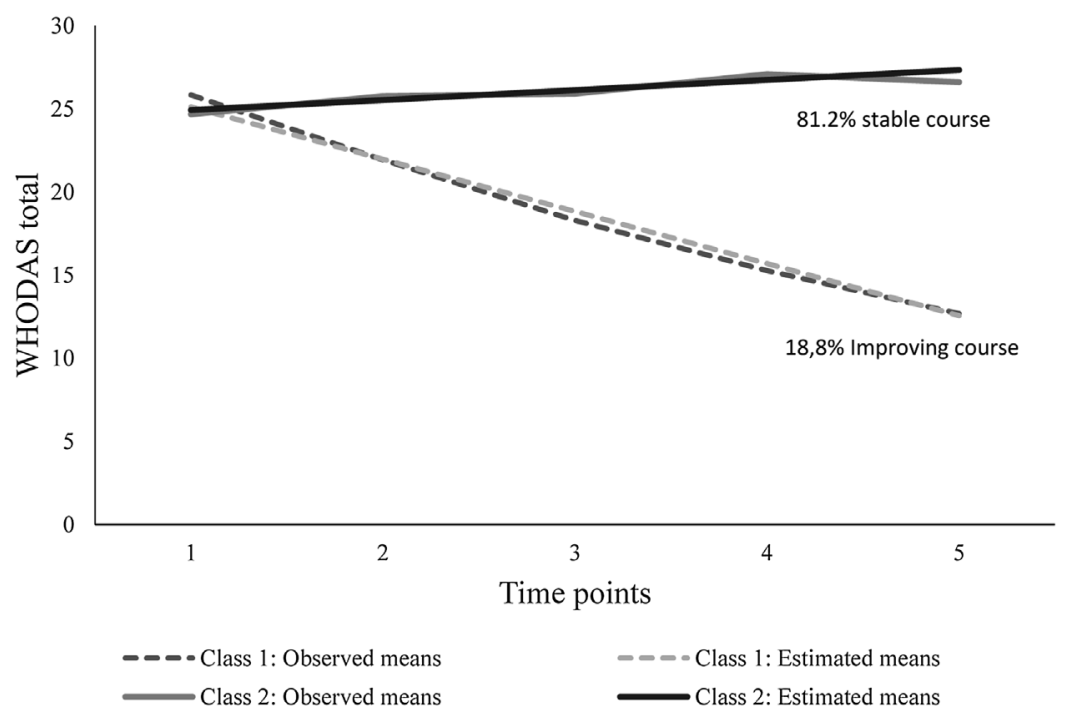

Fig. 1. Growth Mixture Models shows two course types of functional limitations over time.

Table 2

Predictors of no functionnal recovery, adjusted for age, sex, and level of education $(\mathrm{n}=313)^{1}$.

\begin{tabular}{|c|c|c|c|c|}
\hline & Baseline values $^{2}$ & OR & {$[95 \% \mathrm{CI}]$} & $p$ \\
\hline \multicolumn{5}{|l|}{ Socio-demographics: } \\
\hline Age (years) & $70.3(7.3)$ & 1.05 & $1.00-1.09$ & .036 \\
\hline Female sex & $66.5 \%(208 / 313)$ & 1.38 & $0.76-2.52$ & .291 \\
\hline Level of education (years) & $10.6(3.5)$ & 1.08 & $0.99-1.18$ & .083 \\
\hline \multicolumn{5}{|l|}{ Cognitive functioning: } \\
\hline Global cognitive functioning & $28.0(3.0)$ & 1.08 & $0.91-1.28$ & .363 \\
\hline Memory (retention score) & $73.9 .0(1.3)$ & 1.00 & $0.990-1.18$ & .703 \\
\hline Processing speed $^{2}$ & $22.0(5.0)$ & 1.02 & $0.96-1.08$ & .561 \\
\hline Working memory & $13.4(3.2)$ & 1.04 & $0.94-1.45$ & .468 \\
\hline Interference score ${ }^{2}$ & $1.2(0.7)$ & 0.77 & $0.56-1.06$ & .106 \\
\hline \multicolumn{5}{|l|}{ Somatic condition: } \\
\hline Number of chronic diseases & $2.1(1.5)$ & 1.27 & $1.01-1.58$ & .037 \\
\hline Body Mass Index $\left(\mathrm{kg} / \mathrm{m}^{2}\right)$ & $26.2(4.5)$ & 1.04 & $0.97-1.12$ & .251 \\
\hline Total number of medication & $5.6(3.3)$ & 1.03 & $0.94-1.13$ & .579 \\
\hline Handgrip strength (kg) & $28.2(11.4)$ & 0.97 & $0.94-1.01$ & .108 \\
\hline Gait speed (seconds) ${ }^{2}$ & $6.0(3.0)$ & 0.99 & $0.92-1.07$ & .814 \\
\hline \multicolumn{5}{|l|}{ Lifestyle characteristics: } \\
\hline Alcohol use (total score AUDIT) ${ }^{2}$ & $2.0(4.0)$ & 1.08 & $0.97-1.19$ & .157 \\
\hline Current smoker (yes) & $25.7 \%(80 / 311)$ & 1.80 & $0.87-3.72$ & .114 \\
\hline sufficient physical activity & $71.8 \%(219 / 305)$ & 0.93 & $0.47-1.80$ & .818 \\
\hline Sitting minutes per week & $425(190)$ & 1.00 & $1.00-1.00$ & .755 \\
\hline \multicolumn{5}{|l|}{ Psycho-social characteristics: } \\
\hline Partner status, with partner & $54.3 \%(170 / 311)$ & 0.80 & $0.43-1.48$ & .482 \\
\hline Network size (ref $=0-5$ persons) & $58.8 \%(183 / 311)$ & & & \\
\hline middle (6-10 persons) & $27.7 \%(86 / 311)$ & 0.91 & $0.47-1.76$ & .906 \\
\hline large (>10 persons) & $13.5 \%(42 / 311)$ & 0.92 & $0.39-2.20$ & .923 \\
\hline Sense of mastery & $15.7(4.5)$ & 1.03 & $0.97-1.10$ & .347 \\
\hline \multicolumn{5}{|l|}{ Psychopathology: } \\
\hline Depression symptoms & $29.6(13.0)$ & 0.99 & $0.97-1.02$ & .597 \\
\hline Anxiety symptoms & $17.2(11.1)$ & 1.01 & $0.99-1.04$ & .356 \\
\hline
\end{tabular}

Abbreviations: SD: standard deviation; IQR: interquartile range; OR: odds ratio; $\mathrm{CI}$ : confidence interval; p: p-value; AUDIT: Alcohol Use Identification Test.

${ }^{1}$ logistic regression analyses with trajectories of functional limitation ( 0 =improving course, $1=$ stable course) as dependent variable, adjusted for age, gender, education.

2 Baseline values presented as proportion (\% with $\mathrm{n} / \mathrm{N}$ ) or mean (SD), and in case of a skewed distribution as the median (IQR). In case the median is given, this is indicated by $\mathrm{a}^{2}$ after the characteristic in the first column.

\subsection{Course type of functional limitations stratified by remission at follow-up}

Of the 313 included subjects, 272 received a CIDI interview at two-year follow up (as 41 dropped out before the two-year site visit). Therefore, 106 patients from the original sample were excluded. Excluded patients $(n=106)$ did not statistically differ from those included $(n=313)$ with respect with age, sex, years of education, and number of chronic diseases, but had a significantly lower mean MMSE score at baseline $(27.1(\mathrm{SD}=2.5)$ versus 27.9 $(\mathrm{SD}=1.7), \mathrm{t}=-3.6, \mathrm{df}=375, \mathrm{p}<.001)$.

Symptomatic remission of depression was the main predictor of functional recovery. Of the $141 / 272(51.8 \%)$ remitted patients at follow-up, 43/141 (30.5\%) also achieved functional recovery, whereas only $11 / 131(8.4 \%)$ of the non-remitted patients achieved functional recovery $\left(\chi^{2}=20.8, \mathrm{df}=1, \mathrm{p}=<.001\right)$. Subsequently, we explored predictors of no functional recovery stratified by the fact whether patients had achieved a symptomatic remission by multinomial logistic regression (Table 3 ). As only 11 patients achieved functional recovery while not being in symptomatic remission, this subgroup of patients was excluded (sample size too small for multivariable analyses). The results in the final analyse $(\mathrm{n}=261)$ showed that compared to patients with the most optimal results, i.e. symptomatic remission with functional recovery, nonremitted patients without functional recovery are characterized by the presence of more chronic somatic diseases, a lower sense of mastery, and a higher level of anxiety, whereas those patients with a symptomatic remission but no functional recovery are characterized by female sex, higher level of education, higher gait speed, and less severe depression compared to the reference group (see Table 3).

\section{Discussion and conclusions}

\subsection{Main findings}

As expected, depressed patients were functionally significantly more impaired compared to their non-depressed counterparts. Although this study confirms previous findings that depression and functional limitations are highly correlated [33], the most important finding was that only 1 in 5 depressed older patients achieve functional recovery over a period of two years. As expected, the main predictor of functional recovery was symptomatic remission of depression. When stratified for symptomatic remission at two years, 3 in 10 remitted patients also achieve functional recovery, which is still low. Interestingly, 1 in 10 nonremitted patients is able to achieve functional recovery, despite suffering from a chronic depressive disorder. As will be discussed below, the course trajectories of functional limitations and their 
Table 3

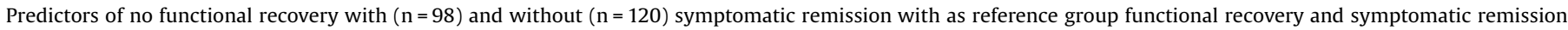
$(n=43)$ adjusted for age, sex and level of education (total $N=261)^{1}$.

\begin{tabular}{|c|c|c|c|c|c|c|}
\hline & \multicolumn{3}{|c|}{ No symptomatic remission } & \multicolumn{3}{|c|}{ Symptomatic remission } \\
\hline & OR & {$[95 \% \mathrm{CI}]$} & $p$ & OR & {$[95 \% \mathrm{CI}]$} & $p$ \\
\hline \multicolumn{7}{|l|}{ Socio-demographics: } \\
\hline Age (years) & 1.05 & $1.00-1.10$ & .075 & 1.04 & $0.99-1.10$ & .153 \\
\hline Female sex & 1.69 & $0.82-3.48$ & .155 & 2.50 & $1.17-5.35$ & .018 \\
\hline Level of education (years) & 1.07 & $0.96-1.19$ & .242 & 1.12 & $1.00-1.26$ & .043 \\
\hline \multicolumn{7}{|l|}{ Cognitive functioning: } \\
\hline Global cognitive functioning & 0.93 & $0.75-1.16$ & .532 & 1.02 & $0.81-1.28$ & .895 \\
\hline Memory (retention score) & 1.00 & $0.99-1.02$ & .981 & 1.01 & $0.99-1.02$ & .551 \\
\hline Processing speed & 1.026 & $0.95-1.10$ & .495 & 0.98 & $0.90-1.06$ & .537 \\
\hline Working memory & 1.03 & $0.14-1.16$ & .619 & 1.05 & $0.92-1.19$ & .471 \\
\hline Interference score & 0.72 & $0.48-1.08$ & .115 & 0.72 & $0.48-1.10$ & .128 \\
\hline \multicolumn{7}{|l|}{ Somatic condition: } \\
\hline Number of chronic diseases & 1.42 & $1.08-1.87$ & .013 & 1.00 & $0.75-1.34$ & .984 \\
\hline Body mass index & 1.07 & $0.98-1.17$ & .112 & 0.96 & $0.88-1.06$ & .421 \\
\hline Total number of medications & 1.07 & $0.95-1.20$ & .254 & 0.95 & $0.84-1.08$ & .427 \\
\hline Handgrip strength (kg) & 0.98 & $0.94-1.02$ & .307 & 0.98 & $0.94-1.02$ & .320 \\
\hline Gait speed (seconds) & 0.97 & $0.88-1.06$ & .499 & 0.88 & $0.79-0.99$ & .034 \\
\hline \multicolumn{7}{|l|}{ Lifestyle characteristics: } \\
\hline Alcohol use (total score AUDIT) & 1.04 & $0.92-1.18$ & .539 & 1.10 & $0.97-1.24$ & .143 \\
\hline Current smoker (yes) & 2.20 & $0.88-5.53$ & .093 & 1.62 & $0.62-4.24$ & .329 \\
\hline sufficient physical activity & 0.82 & $0.35-1.90$ & .638 & 1.17 & $0.48-2.89$ & .727 \\
\hline Sitting minutes per week & 1.00 & $1.00-1.00$ & .892 & 1.00 & $1.00-1.00$ & .216 \\
\hline \multicolumn{7}{|l|}{ Psycho-social characteristics: } \\
\hline Partner status, with partner & 0.66 & $0.31-1.38$ & .267 & 1.16 & $0.53-2.54$ & .708 \\
\hline \multicolumn{7}{|l|}{ Network size (reference: $0-5$ persons) } \\
\hline middle (6-10 persons) & 0.68 & $0.30-1.52$ & .345 & 1.23 & $0.53-2.85$ & .625 \\
\hline large (>10 persons) & 0.75 & $0.21-2.63$ & .650 & 2.90 & $0.88-9.61$ & .082 \\
\hline Sense of mastery & 1.12 & $1.02-1.22$ & .013 & 0.93 & $0.85-1.01$ & .101 \\
\hline \multicolumn{7}{|l|}{ Psychopathology: } \\
\hline Depression symptoms (IDS total score) & 1.02 & $0.99-1.06$ & .139 & 0.94 & $0.91-0.97$ & $<.001$ \\
\hline Anxiety symptoms (BAI total score) & 1.04 & $1.01-1.08$ & .019 & 0.98 & $0.94-1.02$ & .345 \\
\hline
\end{tabular}

Abbreviations: OR, odds ratio; $\mathrm{CI}$, confidence interval; p, p-value; AUDIT, Alcohol Use Identification Test.

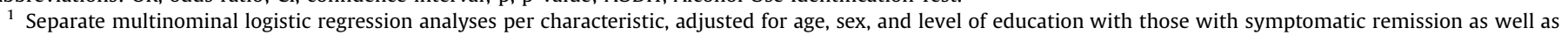
functional recovery $(n=43)$ as the reference group.

determinants might facilitate the development of better, more focused treatment strategies.

\subsubsection{Trajectories towards functional recovery}

To date, the dynamic parallel development of functional limitations and mental health in old age has hardly been explored. One population-based study identified five trajectories of functional limitations and depression symptoms in the last eight years before death. Three groups were identified in which functional limitations and depression were strongly associated. The other two groups of had a medium and high level functional limitations in the absence of any depressive symptoms, pointing the mental health resilience [34]. In contrast to our results in a clinical sample, they did not find a group of well-functioning persons with chronic depressive symptoms.

To our knowledge, only one study has described trajectories of functional recovery in older patients with a depressive disorder [35]. This study identified three classes (each consisting of onethird of the 248 patients) with different trajectories of disability over a four-year follow up. The three trajectories identified were largely determined by the baseline severity of disability. One class was characterized by no disability at all (a class not identified in our study) and the other two trajectories only showed a minor improvement over the first year, but differed by overall severity of disability (low versus high). A high level of disability was associated with female sex, a higher depressive symptom severity at baseline, lower cognitive functioning, and finally a poorer selfrated health. Unfortunately, the course of depression over time was not taken into account. The fact that in this study disability was restricted to a measure of mobile functioning may explain why the three trajectories were in fact relatively stable over time as a change in mobility does not parallel a change in depressive symptom severity over time. By including a broader concept of functional recovery, taking self-reported understanding and communicating, self-care, interpersonal actions, household activities and participation in society into account (in addition to mobility), we found that 2 out of 10 depressed older patients significantly improved over time. Interestingly, neurocognitive functioning was not associated with functional recovery in our study, while neurocognitive dysfunction, especially executive dysfunction and low mental processing speed are associated with persistence of depression symptoms in later life [36]. Moreover, neurocognitive dysfunction has been associated with functional impairment over time among adults with MDD aged up to 65 years $[37,38]$.

\subsubsection{Determinants of functional recovery}

In our study, determinants of non-recovery of functional limitations (irrespective whether a symptomatic remission was achieved) were only a higher age and a higher number of chronic somatic diseases. This suggest that recovery in late-life depression is not determined by modifiable factors. Nonetheless, stratifying results by symptomatic remission yielded a more interesting picture with practical relevance.

Compared to the relatively small group of patients with complete remission and recovery, those without functional recovery could be differentiated by the presence of symptomatic remission. Patients who had achieved a symptomatic remission 
without functional recovery were characterized by a female sex, a higher level of education, a higher gait speed and lower depression severity at baseline. In other words, highly educated and physically healthy women with mild depressive symptoms do remit from their depression but do not recover functionally. Although we have not further characterized this subgroup, one might imagine that these patients suffer from mild comorbid psychiatric disorders, for example personality pathology. Meta-analyses have shown that half of the patients suffering from depression meet criteria for any personality disorder [39]. Previously, we have shown that in NESDO functional limitations are an important confounder in all associations between personality characteristics and depression subtypes and symptom dimensions, suggesting that personality characteristics could be risk factors for depression when facing functional limitations [40]. Future studies should examine psychiatric comorbidity in this specific subgroup as that might also give clues for improving treatment.

Patients who neither achieved a functional recovery nor a symptomatic remission were compared to fully remitted and recovered patients characterized by a higher number of comorbid somatic diseases, a lower sense of mastery and a higher level of anxiety. Interestingly, the combination of chronic somatic disease with a low sense of mastery and high level of anxiety might suggest that coping with physical losses play a part in the chronicity of their depression. Both, the direct effect of somatic comorbidity as well as associated psychosocial adversity, poses older persons at risk for the onset and chronicity of depression [36]. Therefore, this frail and dependent subgroup might profit from integrated care in light of the combined, enduring physical and psychiatric problems. Nurse-led intervention should be put in action to enlarge the sense of mastery and decrease the level of anxiety as nurse-led selfmanagement support for patients with anxiety, depressive or somatic symptoms increase self-efficacy [41].

\subsection{Methodological considerations}

On the one hand, our study has several strengths being one of the first studies on the connection between functional and symptomatic recovery in late life depression. First, we were able to do GMM based on repeated assessment of functional limitations in a relatively large sample of clinically depressed older persons. Furthermore, our results can be generalized to a population of clinically depressed adults that includes different stages of depression and different healthcare settings. Finally, we included many known potential determinants in the relation between functional limitations and depression and were thus able to investigate a comprehensive set of potentially important determinants. Nonetheless, as discussed above, including more determinants like personality characteristics could have been useful. However, taken the scarcity of studies on this topic and the exploratory nature of this study, we had a priori restricted the number of determinants to avoid spurious findings.

On the other hand, several limitations should be taken into account. First of all, patents who dropped out during follow-up had a significantly lower level of cognitive functioning. This might for example explain the lack of any association between cognitive functioning and functional recovery. Secondly, by including a depressed sample at baseline, we have no data on the pre-morbid levels of functional limitations. Functional limitations might be irreversible and an underlying risk factor for the development of depression. If true, this could explain the low rate of functional recovery for at least part of the patients. Second, despite the limitation of multiple testing in objective 3, we chose a level of significance at $\mathrm{p}<0.05$ uncorrected for multiple comparisons. Hereby, it is important to judge P values cautiously, especially when taking the well-acknowledged heterogeneity of MDD into account. Finally, the small group of patients who achieved functional recovery despite a chronic depression is highly interesting. This small subgroup would be interesting to target in future research, as it might increase our knowledge on resilience in late-life depression [42]. Unfortunately, this group was too small to examine in the multivariate analyses.

\subsection{Conclusions}

A growing body of research focusses on the heterogeneity among depressed older patients in an attempt to personalized and improve treatment outcomes $[43,44]$. Combining measures of functional recovery and symptomatic remission may be an important way to disentangle clinically relevant heterogeneity. Although still speculative, our results points to a large group of healthy older patients that might profit from more rigorous psychiatric treatment, which probably should take comorbidity into account, whereas another group of frail depressed older patients may need integrated geriatric rehabilitation. As only 2 out of 10 patients functionally recover, outcome of mental health interventions should be broadened to functional recovery in future studies $[45,46]$ as well as clinical care. In this light, mental health nursing interventions should be incorporated in future treatment studies as these interventions specifically focus on functional recovery.

\section{Declaration of Competing Interest}

None.

\section{Acknowledgements}

The infrastructure for NESDO is funded through the Fonds NutsOhra, Stichting tot Steun VCVGZ, NARSAD The Brain and Behaviour Research Fund, and the participating universities and mental health care organizations (VU University Medical Center, Leiden University Medical Center, University Medical Center Groningen, Radboud University Nijmegen Medical Center, and GGZ inGeest, GGNet, GGZ Nijmegen, GGZ Rivierduinen, Lentis, and Parnassia). The authors maintained full independence in the conduct of this work. The sponsors had no role in design, methods, subject recruitment, data collections, analysis or preparation of the manuscript.

\section{References}

[1] Luppa M, Sikorski C, Luck T, Ehreke L, Konnopka A, Wiese B, et al. Age- and gender-specific prevalence of depression in latest-life-systematic review and meta-analysis. J Affect Disord 2012;136(3):212-21.

[2] Beekman AT, Copeland JR, Prince MJ. Review of community prevalence of depression in later life. Br J Psychiatry 1999;174:307-11.

[3] Kamenov K, Cabello M, Coenen M, Ayuso-Mateos JL. How much do we know about the functional effectiveness of interventions for depression? A systematic review. J Affect Disord 2015;1(188):89-96.

[4] Comijs HC, Nieuwesteeg J, Kok R, van Marwijk HW, van der Mast RC, Naarding $\mathrm{P}$, et al. The two-year course of late-life depression; results from the Netherlands study of depression in older persons. BMC Psychiatry 2015;12(15) 20-015-0401-5.

[5] Hoogendijk EO, Deeg DJ, Poppelaars J, van der Horst M, Broese van Groenou MI, Comijs HC, et al. The Longitudinal Aging Study Amsterdam: cohort update 2016 and major findings. Eur J Epidemiol 2016;31(9):927-45.

[6] Schaakxs R, Comijs HC, Lamers F, Kok RM, Beekman ATF, Penninx BWJH. Associations between age and the course of major depressive disorder: a 2year longitudinal cohort study. Lancet Psychiatry 2018;5(7):581-90.

[7] Karsten J, Hartman CA, Ormel J, Nolen WA, Penninx BW. Subthreshold depression based on functional impairment better defined by symptom severity than by number of DSM-IV symptoms. J Affect Disord 2010;123(13):230-7.

[8] McKnight PE, Kashdan TB. The importance of functional impairment to mental health outcomes: a case for reassessing our goals in depression treatment research. Clin Psychol Rev 2009;(29):243-59. 
[9] Zimmerman M, McGlinchey JB, Posternak MA, Friedman M, Attiullah N, Boerescu D. How should remission from depression be defined? The depressed patient's perspective. Am J Psychiatry 2006;163(1):148-50.

[10] Prodinger B, Cieza A, Oberhauser C, Bickenbach J, Ustun TB, Chatterji S, et al. Toward the international classification of functioning, disability and health (ICF) rehabilitation set: a minimal generic set of domains for rehabilitation as a health strategy. Arch Phys Med Rehabil 2016;97(6):875-84.

[11] Sheehan DV, Nakagome K, Asami Y, Pappadopulos EA, Boucher M. Restoring function in major depressive disorder: a systematic review. J Affect Disord 2017;215:299-313.

[12] van der Voort TY, Seldenrijk A, van Meijel B, Goossens PJ, Beekman AT, Penninx BW, et al. Functional versus syndromal recovery in patients with major depressive disorder and bipolar disorder. J Clin Psychiatry 2015;76(6):e809-14.

[13] Collard RM, Wassink-Vossen S, Schene AH, Naarding P, Verhaak P, Oude Voshaar RC, et al. Symptomatic and functional recovery in depression in later life. Soc Psychiatry Psychiatr Epidemiol 2018;19.

[14] Meltzer H, Bebbington P, Brugha T, McManus S, Rai D, Dennis MS, et al. Physical ill health, disability, dependence and depression: results from the 2007 national survey of psychiatric morbidity among adults in England. Disabil Health J 2012;5(2):102-10.

[15] Kessler RC, Berglund P, Demler O, Jin RMA, Koretz D, Merikangas KR, et al. The epidemiology of major depressive disorder. J Am Med Dir Assoc 2003;280(23).

[16] Ekers D, Murphy R, Archer J, Ebenezer C, Kemp D, Gilbody S. Nurse-delivered collaborative care for depression and long-term physical conditions: a systematic review and meta-analysis. J Affect Disord 2013;149(1-3):14-22.

[17] Comijs HC, van Marwijk HW, van der Mast RC, Naarding P, Oude Voshaar RC, Beekman AT, et al. The Netherlands study of depression in older persons (NESDO); a prospective cohort study. BMC Res Notes 2011;5(4)524-0500-4-524.

[18] Garin O, Ayuso-Mateos JL, Almansa J, Nieto M, Chatterji S, Vilagut G, Validation of the "world health organization disability assessment schedule, WHODAS-2" in patients with chronic diseases 2010;8:. p. 51.

[19] World Health Organization. Composite international diagnostic interview. Version 2.1. Geneva: WHO; 1997.

[20] Folstein MF, Folstein SE, McHugh PR. "Mini-mental state". A practical method for grading the cognitive state of patients for the clinician. J Psychiatr Res 1975;12(3):189-98.

[21] Van der Elst W, van Boxtel MP, van Breukelen GJ, Jolles J. Rey's verbal learning test: normative data for 1855 healthy participants aged 24-81 years and the influence of age, sex, education, and mode of presentation. J Int Neuropsychol Soc 2005;11(3):290-302.

[22] Klein M, Ponds RW, Houx PJ, Jolles J. Effect of test duration on age-related differences in Stroop interference. J Clin Exp Neuropsychol 1997;19(1):77-82.

[23] Walton D. The diagnostic and predictive accuracy of the modified word learning test in psychiatric patients over 65. J Ment Sci 1958;104(437):1119-22.

[24] Kriegsman DM, Penninx BW, van Eijk JT, Boeke AJ, Deeg DJ. Self-reports and general practitioner information on the presence of chronic diseases in community dwelling elderly. A study on the accuracy of patients' self-reports and on determinants of inaccuracy. J Clin Epidemiol 1996;49(12):1407-17.

[25] Patterson E. Guidelines for data processing and analysis of the international physical activity questionnaire (IPAQ). 2005 Available at:https://sites.google. com/site/theipaq/scoring-protocol(Accessed 19 July 2017).

[26] Babor TF, Kranzler HR, Lauerman RJ. Early detection of harmful alcohol consumption: comparison of clinical, laboratory, and self-report screening procedures. Addict Behav 1989;14(2):139-57.
[27] Stansfeld S, Marmot M. Deriving a survey measure of social support: the reliability and validity of the Close Persons Questionnaire. Soc Sci Med 1992;35 (8):1027-35.

[28] Pearlin LI, Schooler C. The structure of coping. J Health Soc Behav 1978;19 (1):2-21.

[29] Andrews G, Peters L. The psychometric properties of the composite international diagnostic interview. Soc Psychiatry Psychiatr Epidemiol 1998;33(2):80-8.

[30] Beck AT, Epstein N, Brown G, Steer RA. An inventory for measuring clinical anxiety: psychometric properties. J Consult Clin Psychol 1988;56:893-7.

[31] Andruff H. Carraro N. Thompson A. Gaudreau P. Latent growth modelling: a tutorial. Tutor Quant Methods Psychol 2009;5(1):11-24.

[32] Muthén LK, Muthén BO. Mplus user’ s guide. Los Angeles CA: Muthén n; 2012.

[33] Verhaak PF, Dekker JH, de Waal MW, van Marwijk HW, Comijs HC. Depression, disability and somatic diseases among elderly. J Affect Disord 2014;167:187-91.

[34] Raab M, Fasang A, Hess M. Pathways to death: the co-occurrence of physical and mental health in the last years of life. Demogr Res 2018:38(53):1619-34.

[35] Hybels CF, Pieper CF, Blazer DG, Fillenbaum GG, Steffens DC. Trajectories of mobility and IADL function in older patients diagnosed with major depression. Int J Geriatr Psychiatry 2010;25(1):74-81.

[36] Tunvirachaisakul C, Gould RL, Coulson MC, Ward EV, Reynolds G, Gathercole RL, et al. Predictors of treatment outcome in depression in later life: a systematic review and meta-analysis. J Affect Disord 2018;227:164-82.

[37] Hammer-Helmich L, Haro JM, Jonsson B, Tanguy Melac A, Di Nicola S, Chollet J, et al. Functional impairment in patients with major depressive disorder: the 2year PERFORM study. Neuropsychiatr Dis Treat 2018;9(14):239-49.

[38] Jaeger J, Berns S, Uzelac S, Davis-Conway S. Neurocognitive deficits and disability in major depressive disorder. Psychiatry Res 2006;145(1):39-48.

[39] Friborg O, Martinsen EW, Martinussen M, Kaiser S, Overgard KT, Rosenvinge JH. Comorbidity of personality disorders in mood disorders: a meta-analytic review of 122 studies from 1988 to 2010. J Affect Disord 2014:152-154:1-11.

[40] Koorevaar AM, Comijs HC, Dhondt AD, van Marwijk HW, van der Mast RC, Naarding P, et al. Big five personality and depression diagnosis, severity and age of onset in older adults. J Affect Disord 2013;151(1):178-85.

[41] Zimmermann T, Puschmann E, van den Bussche H, Wiese B, Ernst A, Porzelt S, et al. Collaborative nurse-led self-management support for primary care patients with anxiety, depressive or somatic symptoms: cluster-randomised controlled trial (findings of the SMADS study). Int J Nurs Stud 2016;63:101-11.

[42] Wermelinger Avila MP, Lucchetti AL, Lucchetti G. Association between depression and resilience in older adults: a systematic review and metaanalysis. Int J Geriatr Psychiatry 2017;32(3):237-46.

[43] Hybels CF, Blazer DG, Landerman LR, Steffens DC. Heterogeneity in symptom profiles among older adults diagnosed with major depression. Int Psychogeriatr 2011;23(6):906-22.

[44] Hybels CF, Pieper CF, Blazer DG, Steffens DC. Heterogeneity in the three-year course of major depression among older adults. Int J Geriatr Psychiatry 2016;31(7):775-82.

[45] IsHak WW, Bonifay W, Collison K, Reid M, Youssef H, Parisi T, et al. The recovery index: a novel approach to measuring recovery and predicting remission in major depressive disorder. J Affect Disord 2017;208:369-74.

[46] Holvast F, Massoudi B, Oude Voshaar RC, Verhaak PFM. Non-pharmacological treatment for depressed older patients in primary care: a systematic review and meta-analysis. PLoS One 2017;12(9)e0184666. 